\title{
Priorização no Balanceamento de Réplicas em Instâncias Heterogêneas do HDFS baseada na Capacidade dos Nodos
}

\author{
Rhauani Weber Aita Fazul ${ }^{1}$, Patrícia Pitthan Barcelos ${ }^{2}$ \\ ${ }^{1}$ Laboratório de Sistemas de Computação (LSC) \\ ${ }^{2}$ Pós-Graduação em Ciência da Computação (PGCC) \\ Universidade Federal de Santa Maria (UFSM) \\ Santa Maria - RS - Brasil \\ \{rwfazul, pitthan\}einf.ufsm.br
}

\begin{abstract}
The HDFS Balancer is an Apache Hadoop daemon that redistributes blocks by moving them across the cluster until the utilization of every node is within a certain threshold. However, the balancer tool is not optimized for HDFS instances running in heterogeneous environments. This work presents a customized balancing policy for HDFS Balancer that allows data distribution based on differences in the capacities of the nodes. Thus, nodes with high capacity are prioritized to receive a high number of replicas during block rearrangement.
\end{abstract}

Resumo. O HDFS Balancer é o balanceador de réplicas nativo do Apache Hadoop, que opera em redistribuir os blocos de dados armazenados no sistema até que a utilização de todos os nodos do cluster fique dentro de um determinado threshold. Entretanto, a ferramenta não é otimizada para o balanceamento de instâncias do Hadoop executando em ambientes heterogêneos. Esse trabalho apresenta uma customização na política de operação padrão do HDFS Balancer que faz com que o reposicionamento das réplicas seja realizado considerando diferenças nas capacidades dos nodos. Dessa forma, nodos com alta capacidade são priorizados para o recebimento de um maior volume de dados.

\section{Introdução}

O HDFS (Hadoop Distributed File System) é o sistema de arquivos distribuído do Apache Hadoaop 1 projetado para ser tolerante a falhas mesmo quando executa em clusters com hardware comum (i.e. não confiável). Um cluster HDFS baseia-se em uma arquitetura mestre-escravo formada por dois tipos de nós: NameNode (NN) e DataNode (DN) [Foundation 2018]. O NN é o servidor mestre que gerencia o namespace e os metadados do sistema e, também, controla o acesso e a distribuição dos arquivos. Enquanto isso, os DNs são os workers que recuperam e armazenam efetivamente os dados.

O HDFS adota uma estrutura de armazenamento própria, otimizada para cenários de big data. Ao ser inserido no sistema, os arquivos são automaticamente segmentados em blocos de dados de tamanho fixo (por padrão 128MB). Ao executar em equipamentos de baixo custo, as chances de falhas de nodo em um cluster HDFS são elevadas [White 2015]. Mesmo com esta condição, o sistema deve garantir alta disponibilidade e confiabilidade, de modo que nenhum bloco seja perdido. Para tal, o HDFS faz uso da replicação de dados: um mecanismo de tolerância a falhas baseado em redundância.

https://hadoop.apache.org/ 
A replicação é essencial para assegurar a integridade e a disponibilidade dos blocos armazenados no sistema, além de ser utilizada como uma estratégia para suprir altas demandas de acesso. A forma de distribuição das réplicas entre os nodos, entretanto, influencia diretamente no balanceamento no cluster. Quando os DNs armazenam quantidades desproporcionais de dados, o desempenho do HDFS é afetado [Fazul et al. 2019]. Uma forma de equilibrar a distribuição de dados no sistema é através do HDFS Balancer, o balanceador nativo do Hadoop. Todavia, a política de operação padrão da ferramenta não é otimizada para instâncias do HDFS executando em ambientes heterogêneos.

Este trabalho apresenta uma customização para o HDFS Balancer sob a forma de uma prioridade de balanceamento. Com a solução proposta, diferenças nas capacidades de armazenamento ou de processamento dos nodos passam a ser consideradas pelo balanceador durante a redistribuição dos dados. Dessa forma, DNs que apresentarem maior capacidade tornam-se prioritários ao recebimento controlado de um maior número de réplicas, enquanto DNs com menor capacidade ficam responsáveis por armazenar um menor volume de dados. Adicionalmente, uma investigação experimental foi conduzida de forma a validar a implementação e analisar possíveis melhorias na localidade dos dados impulsionadas pelo balanceamento de réplicas com base na capacidade dos nodos.

O artigo está organizado em seis seções. A Seção 2 é voltada ao processo de replicação de dados no HDFS. A Seção 3 dedica-se ao balanceamento de réplicas e aos trabalhos relacionados. A Seção 4 detalha a solução desenvolvida. A Seção 5 apresenta e discute os resultados obtidos. Por fim, a Seção 6 aponta as considerações finais.

\section{Replicação de Blocos no HDFS}

A replicação é o principal mecanismo de tolerância a falhas (TF) do HDFS. O mecanismo consiste na criação de cópias dos blocos armazenados no sistema, visando o aumento da confiabilidade e da disponibilidade dos dados por meio de redundância. As réplicas são armazenadas em diferentes DNs, de forma que, em caso de falha de um nodo, os blocos comprometidos possam ser acessados a partir de um ou mais DNs que contenham suas réplicas. A quantidade de réplicas de cada bloco é definida pelo Fator de Replicação (FR).

Além da distribuição inicial dos blocos, é necessário que o NN monitore ativamente o estado das réplicas do sistema, disparando, quando preciso, o processo de rereplicação. A re-replicação é fundamental para a manutenção da confiabilidade do HDFS e, dentre outros motivos, pode ser originada por falhas de DN [Foundation 2018]. Um DN inativo é identificado pelo NN através da ausência de mensagens Hearbeat em um intervalo pré-definido. A partir disto, o FR dos blocos armazenados no DN em questão é decrementado e a re-replicação pode ser disparada pelo NN. Ambos os processos de replicação e re-replicação são realizados de forma transparente pelo HDFS e necessitam de uma decisão do NN: a escolha dos DNs para o armazenamento das réplicas.

Esta escolha deve assegurar a disponibilidade dos blocos em caso de falhas e otimizar o desempenho do HDFS em operações sobre os dados. Para tal, o NN é guiado por uma Política de Posicionamento de Réplicas (PPR), que faz uso de uma estratégia rackaware visando aprimorar a confiabilidade e a TF do sistema com base na arquitetura do cluster [Foundation 2018]. Para um FR padrão de três réplicas por bloco, a PPR faz com que a primeira réplica seja armazenada no DN local (se o cliente HDFS estiver executando fora do cluster o DN é escolhido aleatoriamente) e que as duas réplicas seguintes sejam 
armazenadas em um rack remoto diferente do rack da primeira réplica, porém em dois DNs distintos [White 2015]. Em caso de um FR maior, os DNs adicionais são escolhidos arbitrariamente, porém evitando armazenar muitas réplicas em um mesmo rack.

Para reduzir a sobrecarga de armazenamento das múltiplas réplicas de um mesmo bloco, o HDFS usa um pipeline de replicação [Achari 2015], onde os DNs podem, simultaneamente, receber e encaminhar dados. Assim, permite-se que o processo de escrita seja otimizado e transparente ao cliente, que precisa interagir com apenas um único nodo. Já durante a leitura, o HDFS tira proveito da replicação para suprir altas demandas de acesso. Para tal, as réplicas mais próximas da origem da solicitação são preferidas sobre as réplicas remotas, diminuindo o tempo e o custo gastos com o tráfego de dados. Embora a redundância dos dados em racks distintos aumente a confiabilidade do sistema e permita uma melhor utilização dos recursos computacionais durante operações de entrada e saída (E/S), a PPR não distribui os blocos de forma igualitária entre os DNs [Foundation 2018].

\section{Balanceamento de Réplicas}

Um dos pilares do Hadoop é mover as tarefas de computação para onde estão as réplicas, evitando mover os dados em si e, assim, utilizando o processamento local como forma de evitar o consumo da largura de banda do cluster. Esta funcionalidade é conhecida como data locality [White 2015]. Em um cluster balanceado, é possível explorar a localidade dos dados como forma de aprimorar o desempenho de aplicações voltadas a E/S [Fazul et al. 2019]. Porém, a medida que o desbalanceamento de réplicas se agrava no HDFS, a localidade dos blocos é afetada, podendo ocasionar um maior tráfego off-rack e fazer com que os recursos computacionais não sejam utilizados de forma otimizada.

A PPR garante um balanceamento mínimo, porém favorece o desbalanceamento inter-nodo (DNs são escolhidos arbitrariamente para manter as réplicas, sem considerar a ocupação de cada nodo) e inter-rack (um mesmo rack é selecionado para manter $2 / 3$ das réplicas de um determinado bloco). Além disso, outros aspectos podem promover o desequilíbrio no sistema, tais como: (i) o processo de re-replicação, também sujeito à PPR; (ii) a adição de um DN ao cluster, já que este irá competir igualmente com os demais DNs para o recebimento dos blocos replicados, resultando em um período de subutilização significativo; e (iii) o comportamento da aplicação do cliente que, em função da PPR, armazena uma das réplicas localmente [Hortonworks 2018]. Na Seção 3.1] são apresentadas possíveis abordagens para promover o balanceamento de réplicas no HDFS.

\subsection{Trabalhos Relacionados}

Diferentes estratégias para o balanceamento podem ser encontradas na literatura. Em geral, as soluções podem ser classificadas em proativas ou reativas. Uma solução proativa visa manter o equilíbrio no HDFS através de modificações na PPR. Dessa forma, no momento da distribuição inicial dos blocos, o NN passa a considerar o volume de dados armazenado em cada DN. Exemplos dessa abordagem incluem [Ibrahim et al. 2016], em que os DNs são marcados como livres ou ocupados com base em um histórico da distribuição das réplicas no cluster. Durante a distribuição das réplicas priorizam-se os racks com um maior número de DNs livres. Após, escolhe-se o DN que, estando de acordo com a PPR, possuir o menor espaço de armazenamento ocupado.

Mesmo que as soluções proativas contribuam para manter o HDFS balanceado, nem sempre é possível impedir o desequilíbrio na distribuição de réplicas. Em certas 
situações, como a adição de novos nodos no sistema, o reposicionamento das réplicas já armazenadas torna-se necessário. Exemplos de abordagens reativas para o balanceamento incluem [Liu et al. 2013], que apresentam um algoritmo de balanceamento para equilibrar racks sobrecarregados, visando reduzir as chances de falha total de rack devido à sobrecarga e contribuindo com uma distribuição mais uniforme dos dados. Já [Shah and Padole 2018] focam em otimizar o processo de redistribuição das réplicas aproveitando-se da capacidade de processamento dos nodos, onde os blocos são redistribuídos apenas para DNs específicos, determinados a partir de uma classificação inicial pela heterogeneidade e capacidade de computação de cada DN. Outra possibilidade para o balanceamento reativo é o HDFS Balancer [Shvachko et al. 2010]. Por ser a base para o desenvolvimento deste trabalho, a Seção 3.1.1 é dedicada a este balanceador.

\subsubsection{HDFS Balancer}

O HDFS Balancer [Shvachko et al. 2010] é uma ferramenta do Hadoop dedicada ao balanceamento de réplicas entre dispositivos de armazenamento no HDFS. A ferramenta opera em função de um threshold (por padrão 10\%), que é passado como parâmetro para a execução. Representado como uma porcentagem no intervalo de $0 \%$ a $100 \%$, o threshold limita a diferença máxima que a utilização dos DNs (proporção do espaço em uso no nodo para a capacidade total do nodo) e a utilização geral do cluster (proporção do espaço em uso no cluster para a capacidade total do cluster) pode assumir [White 2015]. Quando a utilização de cada DN estiver dentro desse limite, o cluster é considerado balanceado.

A daemon do HDFS Balancer é disparada sob demanda pelo administrador do sistema e foi projetada para operar sem afetar as demais aplicações em execução no cluster [White 2015]. De todo modo, é possível definir a largura de banda máxima que a ferramenta pode consumir (por padrão $1 \mathrm{MB} / \mathrm{s}$ ). Quanto maior a largura permitida, mais rápido o balanceamento será realizado, porém aumentando as chances de sobrecarga por gerar maior concorrência com os processos do sistema. O fluxo de execução alto-nível do HDFS Balancer pode ser dividido em diferentes etapas realizadas sucessivamente durante a operação da ferramenta [Hortonworks 2018], que são apresentadas a seguir.

O balanceamento inicia com a etapa de classificação dos grupos de dispositivos, onde os dispositivos de armazenamento dos DNs são agrupados de acordo com seus tipos. Sendo $i$ um DN qualquer e $t$ um tipo de dispositivo de armazenamento, considera-se: (i) $G_{i, t}$ como o grupo de dispositivos do tipo $t$ do $\mathrm{DN} i$; (ii) $U_{i, t}$ como a porcentagem que representa a utilização do grupo dos dispositivos do tipo $t$ do $\mathrm{DN} i$; e (iii) $U_{\mu, t}$ como a porcentagem que representa a média de utilização de todos os dispositivos do tipo $t$ do cluster. Os grupos são adicionados em listas globais de acordo com a sua classificação, sendo elas: (i) superutilizado (over-utilized), quando $U_{i, t}>U_{\mu, t}+$ threshold; (ii) acima da média (above-average), quando $U_{i, t}>U_{\mu, t}$ (L. 3); (iii) abaixo da média (below-average), quando $U_{\mu, t} \geq U_{i, t} \geq U_{\mu, t}$ - threshold; (iv) subutilizado (under-utilized), quando $U_{\mu, t}-$ threshold $>U_{i, t}$. A classificação é realizada em um método denominado Balancer.init, que também é responsável por definir, para cada $G_{i, t}$, o volume máximo de dados a ser transferido ou recebido em uma iteração de balanceamento.

Este valor é dado pela variável maxSize2Move, cujo cálculo é apresentado na Figura 1. Por padrão, maxSize2Move equivale ao volume de dados (em bytes) necessário 
para levar a $U_{i, t}$ até a $U_{\mu, t}$. Para os $G_{i, t}$ tidos como destino (utilizationDiff menor que zero), o valor de maxSize2Move é limitado pelo espaço de armazenamento restante no grupo (L. 9). Ao final, garante-se que o valor de maxSize2Move não extrapole o valor definido na propriedade $d f$ s.balancer.max-size-to-move (por padrão 10GB) (L. 11). Se o cluster HDFS não possuir nenhum $G_{i, t}$ subutilizado ou superutilizado ele é considerado balanceado. Caso contrário, a execução do HDFS Bal ancer continua na etapa seguinte.

Figura 1. Cálculo padrão para a definição de maxSize2Move de cada grupo.

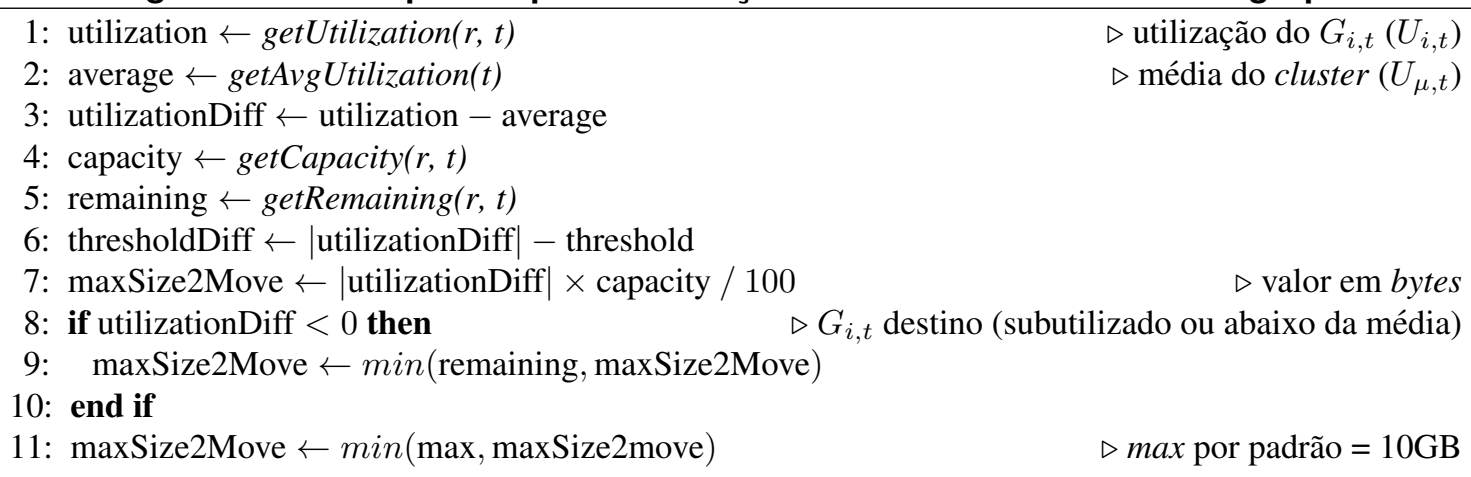

Na etapa de pareamento dos grupos, o método chooseStorageGroups forma pares origem-destino entre os grupos classificados anteriormente. Cada $G_{i, t}$ superutilizado (origem) é pareado com um ou mais $G_{i, t}$ subutilizados (destino) em uma relação $1-N$. Se algum grupo superutilizado possuir maxSize2Move satisfeito, ele é removido da lista e não será mais pareado na iteração corrente. Para os grupos superutilizados remanescentes, são selecionados candidatos nos $G_{i, t}$ classificados como abaixo da média. Se ainda houver algum $G_{i, t}$ subutilizado, procuram-se candidatos entre os $G_{i, t}$ acima da média restantes. A estratégia de pareamento procura, inicialmente, grupos de um mesmo rack. Caso não seja possível formar pares no mesmo rack, aceitam-se grupos de qualquer rack.

No agendamento de movimentação dos blocos, o método dispatchAndCheckContinue inicia uma thread responsável por decidir quais dos blocos da origem devem ser movimentados para o destino. Um bloco do grupo de um DN origem é eletivo ao movimento se: (i) estiver armazenado em um dispositivo do mesmo tipo que o destino; (ii) não estiver em processo de movimentação, nem for um dos blocos já movimentados na iteração; (iii) não possuir uma réplica já existente no destino; e (iv) após a movimentação, continuar em concordância com a PPR. Para diminui o tráfego necessário para a transferência dos dados entre os nodos, o DN mais próximo do destino que possuir uma réplica do bloco a ser movimentado é utilizado como um proxy.

Na etapa de transferência do bloco, o DN destino copia o bloco mantido no DN proxy para seu armazenamento local. Após, ele envia um alerta para o NN, que dispara a deleção da réplica armazenada no DN origem. Concluída a movimentação, a iteração é encerrada e as listas dos grupos são resetadas para as próximas iterações, que serão executadas caso o cluster - dado o threshold configurado - ainda não esteja balanceado.

\section{Política de Balanceamento Customizada}

Estudos passados atestaram que o desbalanceamento de réplicas afeta diretamente o desempenho do HDFS em atender aplicações voltadas a E/S [Fazul et al. 2019]. Motivado 
por isso, definiu-se uma política de balanceamento customizada para o HDFS Balancer que baseia-se em prioridades - primariamente elencadas em [Fazul and Barcelos 2019] dedicadas a flexibilizar e otimizar a operação do balanceador do Hadoop.

Para permitir funcionalidades de priorização na formação de pares de nodos origem-destino durante o balanceamento, a política customizada define um sistema de prioridades com base na topologia do cluster e em diferentes métricas do HDFS. As prioridades implementadas, separadas em categorias de acordo com suas características de funcionamento, são exibidas na Tabela 1 . Todas as prioridades garantem que a disposição dos blocos após o balanceamento permaneça respeitando a PPR e que a variação máxima do volume de dados em cada DN continue sendo controlada pelo valor de threshold. Este trabalho é voltado às prioridades de "capacidade dos nodos", detalhadas na Seção 4.1

Tabela 1. Sistema de prioridades definido pela política customizada.

\begin{tabular}{|l|l|l|}
\hline Categoria & Prioridade & Objetivo geral \\
\hline Capacidade dos nodos & $\begin{array}{l}\text { Capacidade de armazenamento } \\
\text { Capacidade de processamento }\end{array}$ & $\begin{array}{l}\text { Customizar o balanceamento em ambientes heterogêneos a } \\
\text { partir de diferenças de hardware dos DNs. }\end{array}$ \\
\hline Estados dos nodos & $\begin{array}{l}\text { Utilização dos nodos } \\
\text { Classificação dos nodos } \\
\text { Carga dos nodos }\end{array}$ & $\begin{array}{l}\text { Reduzir o impacto da operação do balanceador nas demais } \\
\text { aplicações rodando no HDFS através de priorizações com } \\
\text { base em métricas recuperadas em tempo de execução. }\end{array}$ \\
\hline Estados dos racks & $\begin{array}{l}\text { Confiabilidade dos racks } \\
\text { Utilização dos racks }\end{array}$ & $\begin{array}{l}\text { Permitir que o balanceamento seja conduzido considerando } \\
\text { características dos racks que agrupam os DNs do } \text { cluster. }\end{array}$ \\
\hline Distribuição dos dados & Disponibilidade dos dados & $\begin{array}{l}\text { Priorizar movimentações de réplicas que permitam } \\
\text { aumentar a disponibilidade final dos blocos armazenados. }\end{array}$ \\
\hline
\end{tabular}

\subsection{Priorização baseada na Capacidade dos Nodos}

As prioridades de capacidade são dedicadas a instâncias do Hadoop executando em ambientes heterogêneos. De acordo com as diferenças de hardware das máquinas que rodam cada DN, a estratégia de reposicionamento das réplicas do HDFS Balancer é modificada. Os critérios para a priorização podem basear-se nas capacidades de processamento ou de armazenamento dos nodos. Ambas são similares em quesitos de implementação, diferindo apenas nas métricas utilizadas para determinar os nodos suscetíveis ao recebimento de uma maior ou menor quantidade de dados. A prioridade de capacidade de processamento baseia-se em diferenças na capacidade de computação dos DNs (quantidade de memória RAM). Já a prioridade de capacidade de armazenamento leva em consideração diferenças na capacidade de armazenamento dos DNs (total, utilizada e remanescente). Neste trabalho, o termo "capacidade", quando não especificado, varia de acordo com a prioridade configurada na etapa de balanceamento.

Para incorporar as priorizações, duas diretrizes devem ser seguidas: (i) um nodo com baixa capacidade deve ter prioridade em transferir dados; e (ii) um nodo com alta capacidade deve ter prioridade em receber dados. A primeira modificação a ser realizada no código-fonte do balanceador diz respeito à ordenação das listas responsáveis por manter os grupos de dispositivos de armazenamento dos nodos. Essas listas são separadas a partir da classificação dos $G_{i, t}$ realizada pelo balanceador. De modo a alcançar a diretriz (i), as listas dos grupos origem na transferência dos blocos (superutilizados e acima da média) devem ser ordenadas de forma decrescente baseadas na capacidade dos nodos (seja de processamento ou de armazenamento). Já para atingir a diretriz (ii), as listas dos grupos destino na redistribuição dos blocos (subutilizados e abaixo da média) devem ser ordenadas de forma crescente, também baseadas na capacidade dos nodos. 
A segunda atualização está relacionada ao cálculo do volume de dados máximo permitido que pode ser movimentado do $G_{i, t}$ origem para o $G_{i, t}$ destino em cada iteração de balanceamento. Esse valor é determinado pela variável maxSize2Move. Com a política padrão do HDFS Balancer, maxSize2Move - cujo cálculo foi apresentado na Figura 1 da Seção 3.1.1 - representa a quantidade de dados necessária para levar a utilização de um determinado grupo de dispositivos $\left(U_{i, t}\right)$ até a utilização média dos dispositivos de armazenamento tipo $t$ do cluster $\left(U_{\mu, t}\right)$. Com as modificações realizadas, a variável maxSize2Move passa a ser definida seguindo as diretrizes (i) e (ii).

Para tal, foi implementado um método dedicado à quantificação das capacidades de cada $G_{i, t}$ de acordo com a prioridade configurada. A Figura 2 apresenta o algoritmo definido no método computeWeight. Como somente é preciso executá-lo uma vez a cada iteração de balanceamento, a invocação do novo método foi incorporada em um momento anterior à chamada do método (padrão do balanceador) Balancerinit.

Figura 2. Método responsável por quantificar a capacidade de cada grupo.

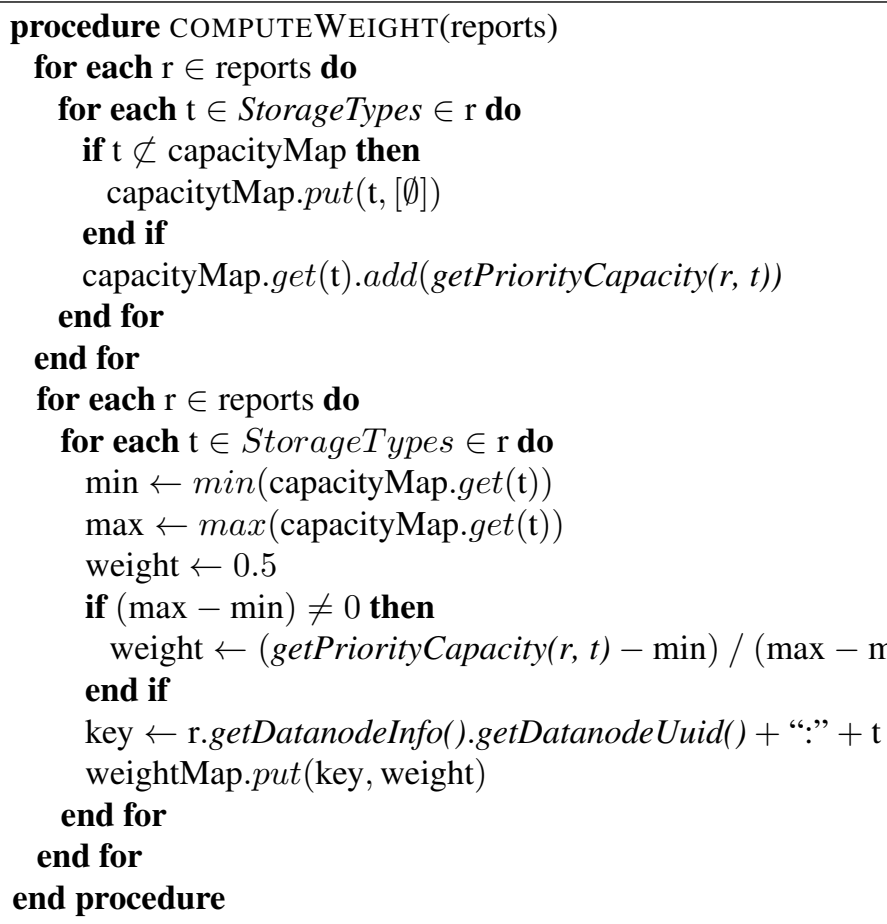

Na primeira parte do método computeWeight (L. 2 a 9), é preenchida a estrutura capacityMap, que relaciona cada tipo de dispositivo $t$ com as capacidades totais dos grupos daquele tipo (a acumulação é realizada pelo método add na linha 7). O método auxiliar getPriorityCapacity retorna um valor que representa a capacidade do grupo de acordo com a prioridade configurada $\left(C_{i, t}\right)$. Para a prioridade de capacidade de armazenamento, esse método irá realizar uma chamada ao método getCapacity (já utilizado pelo balanceador padrão), que devolve o valor em bytes referente a capacidade de armazenamento do $G_{i, t}$. Já para a prioridade de capacidade de processamento, o valor retornado equivale à capacidade de memória RAM total do nodo. Para estender a política customizada de modo a oferecer suporte a novas prioridades de capacidade, além de armazenamento e processamento (e.g. prioridade que considere características dos processadores de cada nodo), bastaria adicionar o retorno adequado em getPriorityCapacity. 
Na segunda parte do método (L. 10 a 21), é criada a estrutura weightMap, que registra, para cada $G_{i, t}$, um valor que representa sua capacidade $\left(C_{i}^{\prime}\right)$. Para tal, aplica-se a normalização min-max em função da capacidade original de cada grupo $\left(C_{i, t}\right)$, de modo que $C_{i}^{\prime}=\left(C_{i, t}-\min \right) /(\max -\min )$. A normalização min-max torna o valor mínimo de um conjunto de valores em 0 , o valor máximo em 1 e, qualquer outro valor, em um decimal entre 0 e 1 . Caso os $G_{i, t}$ não apresentem diferenças de capacidade, $C_{i}^{\prime}$ terá um valor médio de 0,5 (L. 14), fazendo com que a posterior definição de maxSize2Move seja similar ao cálculo atual adotado pela política padrão do balanceador $\left(\left|U_{i, t}-U_{\mu, t}\right|\right)$.

Após computar a $C_{i}^{\prime}$ de cada $G_{i, t}($ L. 16) e definir uma chave que representa o grupo (L. 18), cria-se uma entrada na estrutura weightMap (L. 19). Esta estrutura é posteriormente acessada pelo método calcMaxSize2MoveBasedOnNodeCapacity (Figura 3), que foi implementado de forma a aumentar ou diminuir o valor de maxSize2Move - respeitando a propriedade $d f$ s.balancermax-size-to-move - com base na $C_{i}^{\prime}$ de cada grupo. Sua chamada foi incorporada ao método Balancer.init, de modo que quando o HDFS Balancer for executado com alguma prioridade, o cálculo padrão de maxSize2Move seja substituído pela variação implementada na política customizada. Ressalta-se que o parâmetro capacity desse método refere-se à capacidade de armazenamento do $G_{i, t}$ (não confundir com a capacidade normalizada $-C_{i}^{\prime}-$ armazenada no weightMap), sendo necessária para o cálculo independente da prioridade de capacidade configurada.

Figura 3. Método dedicado ao cálculo customizado da variável maxSize2Move.

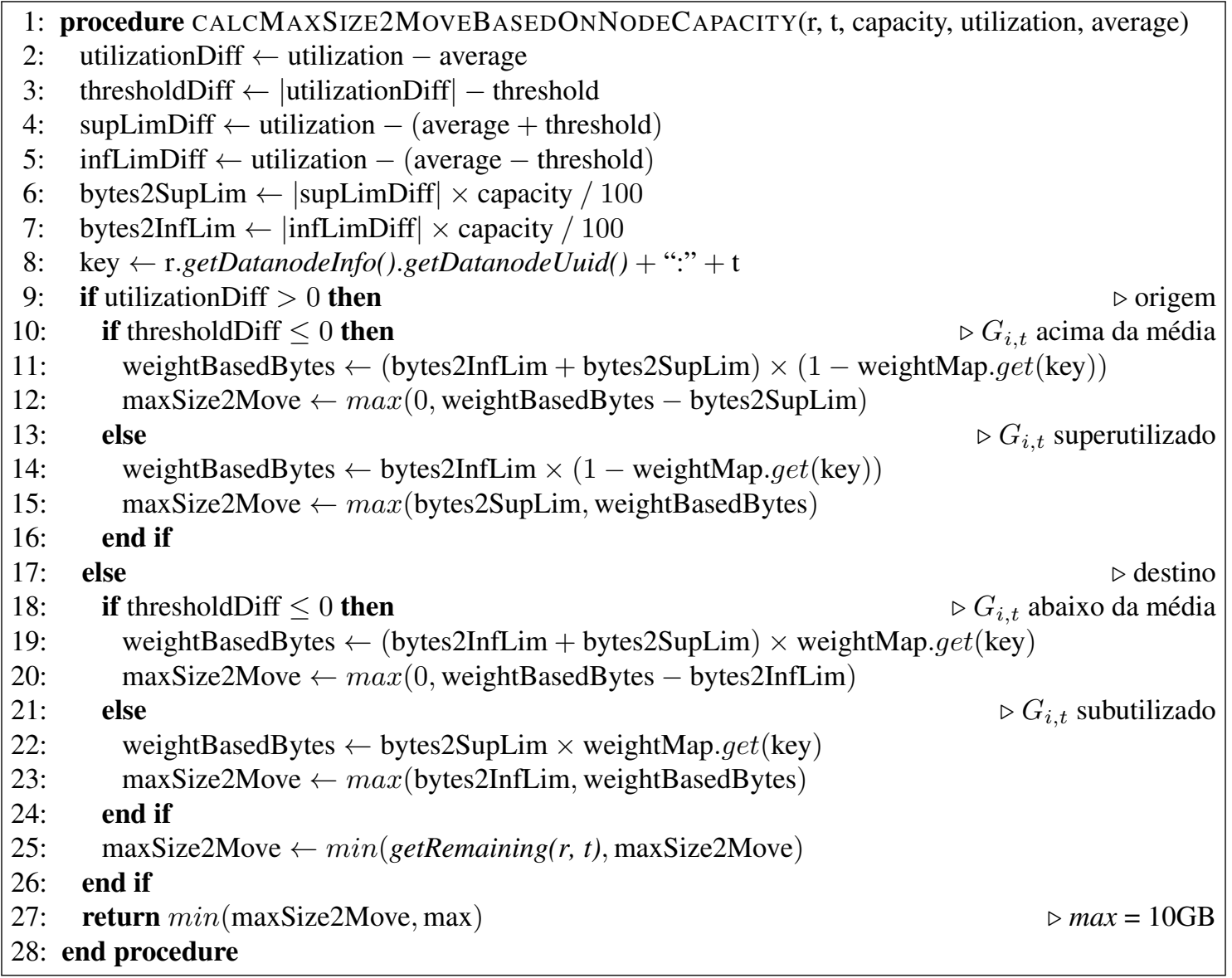

Inicialmente, para cada $G_{i, t}$, calcula-se a diferença de sua utilização $\left(U_{i, t}\right)$ para 
o limite superior $\left(U_{\mu, t}+\right.$ threshold $)$ e para o limite inferior $\left(U_{\mu, t}-\right.$ threshold $)$ que são considerados pelo balanceador (L. 4 e 5). Esses valores, por sua vez, permitem definir a quantidade de bytes necessária para levar a $U_{i, t}$ de um grupo até o limite máximo para que este seja considerado como acima ou abaixo da média (L. 6 e 7). Em seguida, o valor de maxSize2Move é definido de acordo com a classificação e a $C_{i}^{\prime}$ do grupo. De forma geral, o valor da variável maxSize2Move para um $G_{i, t}$ com uma $C_{i}^{\prime}$ elevada aproxima-se do volume de bytes necessário para elevar sua $U_{i, t}$ até o limite superior. Já para um $G_{i, t}$ com uma $C_{i}^{\prime}$ baixa, aproxima-se do volume de bytes necessário para reduzir sua $U_{i, t}$ até o limite inferior. Para idealizar este comportamento, é importante observar alguns detalhes. Em um grupo origem, maxSize2Move determina a redução máxima de sua $U_{i, t}$ (i.e. não é possível aumentar sua utilização para um valor superior à $U_{i, t}$ atual). Analogamente, para um grupo destino, maxSize 2 Move determina a extensão máxima de sua $U_{i, t}$ (i.e. não é possível reduzir sua utilização para um valor inferior à $U_{i, t}$ atual).

Sendo assim, se a $C_{i}^{\prime}$ de um $G_{i, t}$ classificado como acima da média resultar em uma utilização superior à $U_{i, t}$ atual do grupo, maxSize2Move deverá ter o valor zero (L. 12), garantindo assim que sua utilização não seja reduzida. Similarmente, em um $G_{i, t}$ abaixo da média, se sua $C_{i}^{\prime}$ resultar em uma utilização inferior à $U_{i, t}$ atual do grupo, maxSize2Move também deverá ter o valor zero (L. 20), garantindo que sua utilização não seja aumentada. Além disso, em grupos superutilizados com alta $C_{i}^{\prime}$, o valor de maxSize2Move deve, ao mínimo, ser o suficiente para permitir a classificação do grupo como acima da média (garantido pelo método max na linha 15). Já em grupos subutilizados com baixa $C_{i}^{\prime}$, o valor de maxSize2Move deve, ao mínimo, ser o suficiente para permitir a classificação do grupo como abaixo da média (garantido pelo método max na linha 23).

Por fim, são aplicadas duas validações (definidas também no cálculo realizado pela política padrão): (i) em grupos destino, maxSize2Move deve ser inferior ao espaço de armazenamento remanescente no $G_{i, t}$ (L. 25); e (ii) maxSize2Move deve respeitar o valor de max (dado pela propriedade $d f$ s.balancer.max-size-to-move) (L. 27).

\section{Experimentação}

Os experimentos foram realizados na plataforma GRID'50002 $2^{2}$ com o Hadoop (versão 2.9.2) em modo de operação totalmente distribuído. Tendo em vista que as prioridades de capacidade são voltadas a ambientes heterogêneos, o HDFS foi configurado com múltiplos racks contendo nodos com diferenças de hardware entre si.

Ao total, foram configurados 24 nodos dispostos em 3 racks lógicos distintos $\left(R_{1}\right.$, $R_{2}$ e $R_{3}$ ) do site Rennes, onde cada rack executou, respectivamente, 8,6 e 10 DNs e cada DN mantinha um único dispositivo de armazenamento do tipo DISK. Os DNs do rack $R_{1}$ pertenciam ao cluster paravance e cada um possuía 2 processadores Intel Xeon E5-2630 v3 (8 cores por CPU), 128GB de memória RAM e 509,68GB de capacidade de armazenamento, com duas conexões Ethernet de 10Gbps. Os DNs do rack $R_{2}$, pertencentes ao cluster parapide, possuíam 2 processadores Intel Xeon X5570 (4 cores por CPU), 24GB de memória RAM e 417,99GB de capacidade de armazenamento, com uma conexão InfiniBand de 20Gbps. Já os DNs do rack $R_{3}$, pertencentes ao cluster parapluie, possuíam

\footnotetext{
${ }^{2}$ Grid'5000 é uma plataforma para experimentos apoiada por um grupo de interesses científicos hospedado pelo Inria e incluindo CNRS, RENATER e diversas Universidades, bem como outras organizações (mais detalhes em https://wWw.grid5000.fr)
} 
2 processadores AMD Opteron 6164 HE (12 cores por CPU), 48GB de memória RAM, 188,77GB de capacidade de armazenamento e conexão InfiniBand de 20Gbps.

Devido às configurações do ambiente de testes, a prioridade de balanceamento avaliada nesta seção refere-se à capacidade de armazenamento dos nodos. Assim, a normalização min-max foi aplicada com base no espaço de disco total dos DNs $\left(C_{i, D I S K}\right)$. As capacidades normalizadas $\left(C_{i}^{\prime}\right)$ para os DNs dos racks $R_{1}, R_{2}$ e $R_{3}$ foram de, respectivamente, 1,0, 0,7 e 0,0. Para avaliar a distribuição dos blocos, 10 arquivos de 40GB com um FR de 3 réplicas por bloco foram escritos no HDFS através do benchmark TestDFSIO [White 2015], totalizando um volume de dados de 1,2TB.

\subsection{Resultados e Discussão}

Ao total, o HDFS possuía um espaço de armazenamento de 8,27TB estando com 1,22TB ocupados $\left(U_{\mu, D I S K}\right.$ em 14,75\%). Inicialmente, o rack $R_{1}$ mantinha 828,93GB dos dados (utilização em 20,33\%), o rack $R_{2}$ mantinha 289,46GB (utilização em 11,54\%) e o rack $R_{3}$ mantinha $129,5 \mathrm{~GB}$ (utilização em $6,86 \%$ ). A Tabela 2 apresenta a ocupação $\left(O_{i, t}\right)$ e a porcentagem de utilização $\left(U_{i, t}\right)$ de cada $\mathrm{DN}$ sem balanceamento de réplicas (distribuição dos dados inicial, baseada na PPR) e após a execução do HDFS Bal ancer configurado com a prioridade de capacidade de armazenamento e um threshold de $5 \%$.

\section{Tabela 2. Estado do HDFS antes e após o balanceamento de réplicas com a prioridade de capacidade de armazenamento dos nodos.}

\begin{tabular}{|c|c|c|c|c|c|c|c|}
\hline \multirow[b]{2}{*}{ Rack } & \multirow[b]{2}{*}{$\mathrm{C}_{\mathbf{i}, \text { DISK }}$} & \multirow[b]{2}{*}{$\mathbf{C}_{\mathrm{i}}^{\prime}$} & \multirow[b]{2}{*}{ DataNode } & \multicolumn{2}{|c|}{ s/ balanceamento } & \multicolumn{2}{|c|}{ c/ balanceamento } \\
\hline & & & & $\begin{array}{c}O_{i, D I S K} \\
(\mathrm{~GB})\end{array}$ & $\begin{array}{c}U_{i, D I S K} \\
(\%)\end{array}$ & $\begin{array}{c}O_{i, D I S K} \\
(\mathrm{~GB})\end{array}$ & $\begin{array}{c}U_{i, D I S K} \\
(\%)\end{array}$ \\
\hline \multirow{8}{*}{$R_{1}$} & \multirow{8}{*}{509,68} & \multirow{8}{*}{1,0} & $\mathrm{DN}_{01}$ & 137,56 & 26,99 & 96,89 & 19,01 \\
\hline & & & $\mathrm{DN}_{02}$ & 127,47 & 25,01 & 96,89 & 19,01 \\
\hline & & & $\mathrm{DN}_{03}$ & 109,43 & 21,47 & 96,89 & 19,01 \\
\hline & & & $\mathrm{DN}_{04}$ & 108,51 & 21,29 & 96,89 & 19,01 \\
\hline & & & $\mathrm{DN}_{05}$ & 111,11 & 21,80 & 96,89 & 19,01 \\
\hline & & & $\mathrm{DN}_{06}$ & 81,85 & 16,06 & 81,85 & 16,06 \\
\hline & & & $\mathrm{DN}_{07}$ & 76,50 & 15,01 & 76,50 & 15,01 \\
\hline & & & $\mathrm{DN}_{08}$ & 76,50 & 15,01 & 76,50 & 15,01 \\
\hline \multirow{6}{*}{$R_{2}$} & \multirow{6}{*}{417,99} & \multirow{6}{*}{0,7} & $\mathrm{DN}_{09}$ & 83,26 & 19,92 & 62,11 & 14,86 \\
\hline & & & $\mathrm{DN}_{10}$ & 0,00 & 0,00 & 46,10 & 11,03 \\
\hline & & & $\mathrm{DN}_{11}$ & 97,77 & 23,39 & 62,24 & 14,89 \\
\hline & & & $\mathrm{DN}_{12}$ & 54,17 & 12,96 & 55,63 & 13,31 \\
\hline & & & $\mathrm{DN}_{13}$ & 54,26 & 12,98 & 55,38 & 13,25 \\
\hline & & & $\mathrm{DN}_{14}$ & 0,00 & 0,00 & 45,35 & 10,85 \\
\hline \multirow{10}{*}{$R_{3}$} & \multirow{10}{*}{188,77} & \multirow{10}{*}{0,0} & $\mathrm{DN}_{15}$ & 21,26 & 11,26 & 21,26 & 11,26 \\
\hline & & & $\mathrm{DN}_{16}$ & 0,00 & 0,00 & 18,67 & 9,89 \\
\hline & & & $\mathrm{DN}_{17}$ & 44,04 & 23,33 & 27,99 & 14,83 \\
\hline & & & $\mathrm{DN}_{18}$ & 0,00 & 0,00 & 18,67 & 9,89 \\
\hline & & & $\mathrm{DN}_{19}$ & 0,00 & 0,00 & 18,71 & 9,91 \\
\hline & & & $\mathrm{DN}_{20}$ & 23,12 & 12,25 & 23,12 & 12,25 \\
\hline & & & $\mathrm{DN}_{21}$ & 13,61 & 7,21 & 17,82 & 9,44 \\
\hline & & & $\mathrm{DN}_{22}$ & 13,86 & 7,34 & 18,74 & 9,93 \\
\hline & & & $\mathrm{DN}_{23}$ & 13,61 & 7,21 & 18,74 & 9,93 \\
\hline & & & $\mathrm{DN}_{24}$ & 0,00 & 0,00 & 18,86 & 9,99 \\
\hline
\end{tabular}

O rack $R_{1}$ possuía 5 nodos superutilizados $\left(\mathrm{DN}_{01}\right.$ a $\left.\mathrm{DN}_{05}\right)$, i.e. com utilização acima de $19,75 \%\left(U_{\mu, D I S K}+\right.$ threshold $)$. Tendo em vista a capacidade normalizada $\left(C_{i}^{\prime}\right)$ dos nodos do rack em questão $(1,0)$, a utilização dos DNs superutilizados após o balanceamento ficou próxima ao limite superior. Já os demais DNs desse rack $\left(\mathrm{DN}_{06}\right.$ a $\left.\mathrm{DN}_{08}\right)$ estavam classificados como acima da média. Dessa forma, conforme citado durante o relato da implementação dessa prioridade, a utilização desses nodos não poderia ser estendida 
para além da atual. Sendo assim, dada a $C_{i}^{\prime}$ em 1,0, o volume de dados armazenado nos DNs com utilização acima da média foi mantido inalterado.

Em relação ao rack $R_{2}$, haviam 2 nodos subutilizados $\left(\mathrm{DN}_{10}\right.$ e $\left.\mathrm{DN}_{14}\right)$, com utilização abaixo de $9,75 \%$ (U $U_{\mu, D I S K}$ - threshold), 3 nodos classificados como abaixo da média $\left(\mathrm{DN}_{09}, \mathrm{DN}_{12}\right.$ e $\left.\mathrm{DN}_{13}\right)$ e 1 único nodo superutilizado $\left(\mathrm{DN}_{11}\right)$. Dada a $C_{i}^{\prime}$ dos nodos desse rack $(0,7)$, suas utilizações foram levadas, na medida do possível, para próximo da $U_{\mu, D I S K}$. Como nem sempre há um volume de dados suficiente a ser transferido por outros nodos do cluster, a utilização de determinados DNs desse rack $\left(\mathrm{DN}_{10}\right.$ e $\left.\mathrm{DN}_{14}\right)$ ficou abaixo do que seria esperado em função de suas capacidades normalizadas.

Os DNs do rack $R_{3}$, por sua vez, possuíam a menor $C_{i}^{\prime}$ do cluster $(0,0)$. Assim, a prioridade de capacidade esforça-se em reduzir ao máximo suas utilizações. Após o balanceamento, todos os nodos subutilizados desse rack ficaram com utilização próxima ao limite inferior (9,75\%). Já os DNs classificados como abaixo da média $\left(\mathrm{DN}_{15}\right.$ e $\left.\mathrm{DN}_{20}\right)$ e com $C_{i}^{\prime}$ em 0,0 , não poderiam ter sua utilização reduzida para além da atual e, dessa forma, o volume de dados nestes nodos foi mantido inalterado. De forma similar, o $\mathrm{DN}_{17}$ (superutilizado) ficou com utilização próxima a $U_{\mu, D I S K}$ (dado que, no momento que sua $U_{i, t}$ ficasse abaixo da $U_{\mu, D I S K}$, ele estaria na mesma situação que o $\mathrm{DN}_{15}$ e o $\mathrm{DN}_{20}$ ).

Tendo as capacidades normalizadas dos nodos com base no agrupamento por rack, onde $C_{R_{1}}^{\prime}>C_{R_{2}}^{\prime}>C_{R_{3}}^{\prime}$, observa-se que o rack cujos nodos possuíam a maior capacidade calculada $\left(R_{1}\right)$ ficou responsável por manter um maior volume de dados $(719,3 \mathrm{~GB}$ ao total e utilização em $17,64 \%)$ que o rack com a segunda maior capacidade $\left(R_{2}\right)(326,81 \mathrm{~GB}$ e utilização em 13,03\%). Enquanto isso, o rack com menor capacidade $\left(R_{3}\right)$, ficou com a menor ocupação dentre os demais (162,65GB e utilização de 10,78\%), validando assim o objetivo da prioridade de capacidade de armazenamento dos nodos.

De forma a avaliar o impacto do balanceamento com a prioridade de capacidade de armazenamento no desempenho do sistema, mediu-se o tempo necessário para a leitura dos dados com o benchmark TestDFSIO. A Figura 4 exibe os tempos de 20 execuções distintas do benchmark. Inicialmente, o tempo médio de leitura foi de 950,18 segundos. Com o balanceamento de réplicas, esse valor foi reduzido para 806,83 segundos. Considerando a variação percentual dada por $\left(\left(T_{b}-T_{a}\right) / T_{a} \times 100\right)$, onde $T_{a}$ e $T_{b}$ equivalem, respectivamente, às médias aritméticas dos tempos de execução nas 20 execuções do benchmark antes e após a execução do balanceador, a variação alcançada foi de $-15,09 \%$, indicando a redução no tempo necessário para a leitura dos dados.

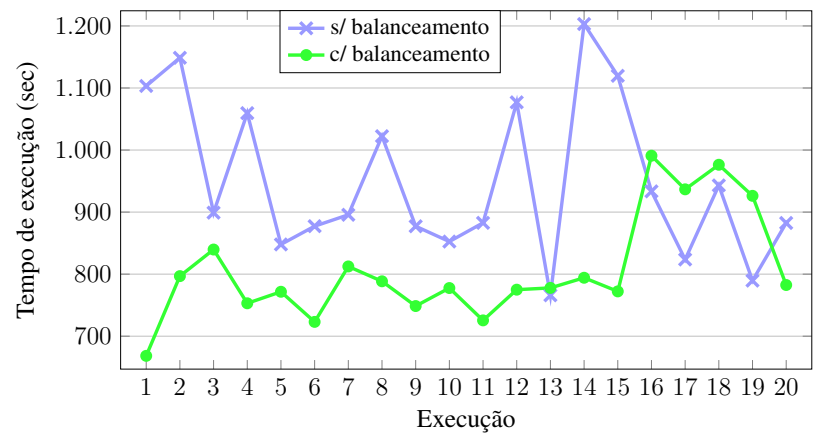

Figura 4. Tempo para leitura dos dados antes e após o balanceamento com a prioridade de capacidade de armazenamento dos nodos. 


\section{Considerações Finais}

Este trabalho apresentou uma estratégia de balanceamento de réplicas customizada para o HDFS Balancer baseada em prioridades de capacidade, que são otimizadas para instâncias do Hadoop executando em ambientes heterogêneos. A solução proposta visa flexibilizar a operação do balanceador nativo do HDFS de forma que diferenças nas capacidades de armazenamento ou de processamento sejam utilizadas como forma de priorização dos nodos para o recebimento de um maior ou menor volume de dados durante o balanceamento do cluster. Após detalhar o funcionamento das prioridades, foram conduzidos experimentos voltados a validar e avaliar a efetividade de implementação.

Os resultados demostram que a priorização do balanceamento do HDFS em função das diferenças nas capacidades dos nodos permite uma exploração otimizada da localidade dos dados, aprimorando o desempenho geral do sistema de arquivos do Hadoop em atender operações de entrada e saída. Trabalhos futuros envolvem uma investigação aprofundada das prioridades em ambientes que permitam endereçar demandas de uso específicas de aplicações reais. Em complemento, pretende-se estender a implementação de modo a permitir que as prioridades de capacidade de armazenamento e de processamento dos nodos possam ser utilizadas de forma associada pelo HDFS Balancer.

\section{Referências}

Achari, S. (2015). Hadoop Essentials. Packt Publishing Ltd, Birmingham, 1st edition.

Fazul, R. W. A. and Barcelos, P. P. (2019). Política customizada de balanceamento de réplicas para o hdfs balancer do apache hadoop. In Anais do XX Workshop de Testes e Tolerância a Falhas, pages 93-106. SBC.

Fazul, R. W. A., Cardoso, P. V., and Barcelos, P. P. (2019). Análise do impacto da replicação de dados implementada pelo apache hadoop no balanceamento de carga. In Anais do X Computer on the Beach, pages 579-588, Florianópolis. Univali.

Foundation, A. S. (2018). "HDFS Architecture". hadoop. apache.org/docs/r2. 9.2/hadoop-project-dist/hadoop-hdfs/HdfsDesign. Junho.

Hortonworks (2018). "HDFS Administration". https://docs.hortonworks. com/HDPDocuments/HDP 2/HDP-2.6.5/bk_hdfs-administration/ content/ch_balancing-in-hdfs.html, Junho.

Ibrahim, I. A., Dai, W., and Bassiouni, M. (2016). Intelligent data placement mechanism for replicas distribution in cloud storage systems. In IEEE International Conference on Smart Cloud (SmartCloud), pages 134-139, New York. IEEE.

Liu, K., Xu, G., and Yuan, J. (2013). An improved hadoop data load balancing algorithm. Journal of Networks, 8(12):2816-2822.

Shah, A. and Padole, M. (2018). Load balancing through block rearrangement policy for hadoop heterogeneous cluster. In 2018 Int. Conference on Advances in Computing, Communications and Informatics (ICACCI), pages 230-236, Bangalore. IEEE.

Shvachko, K., Kuang, H., Radia, S., and Chansler, R. (2010). The hadoop distributed file system. In Symposium on Mass Storage Systems and Technologies, pages 1-10. IEEE.

White, T. (2015). Hadoop: The Definitive Guide. O'Reilly Media, Inc., 4 edition. 